\title{
ULTRASPHERICAL POLYNOMIALS APPLIED TO NONLINEAR VIBRATIONS OF CONTINUOUS MEDIA*
}

\author{
By P. T. BLOTTER (Utah State University) \\ AND D. H. Y. YEN (Michigan State University)
}

Introduction. In several recent papers [1-5] a method for treating discrete nonlinear vibration problems has been developed. By linearizing the nonlinear forces using a set of ultraspherical polynomials over the interval in which the motion takes place, approximate nonlinear amplitude-frequency relations with fair accuracy are obtained from the solutions of the resulting linear systems. Some more general linearizations have also been reported $[6,7]$. The method is restricted essentially to systems which have one degree of freedom or may be characterized by a single amplitude [8].

In this note, the method of ultraspherical polynomials is extended to systems governed by nonlinear partial differential equations to obtain approximate nonlinear amplitude-frequency relations in the neighborhood of the linear eigenvibrations. It is assumed that the systems depend on one space variable and time, and the nonlinear terms in the equations depend on the displacement and its spatial derivatives but not on time explicitly. An obvious difficulty immediately arises when one attempts to follow the method developed for discrete systems because the amplitude of motion is a function of the space variable and not known in advance. To overcome this difficulty some appropriate "mode of deflection" must be assumed. In cases where a linear mode is known, it is taken to be the mode of deflection; otherwise some suitable approximation to the linear mode must be made. Next, an amplitude parameter is introduced so that the amplitude of motion is given by the product of the amplitude parameter and the normalized linear mode. Using ultraspherical polynomials, the nonlinear terms are replaced by terms that are linear in the displacement and linear partial differential equations result. With the given homogeneous boundary conditions one is led to solve linear eigenvalue problems. The determination of the eigenvalues leads to the desired amplitudefrequency relations.

The method. The governing equations in dimensionless form are taken as

$$
L_{x} u+\omega^{2} u_{t t}+N_{x} u=0
$$

where $L_{x}$ is a linear differential operator of order $2 n, u$ is a function of the space variable $x$ and time $t, \omega$ is a frequency parameter, and $N_{x} u$ is a nonlinear function of $u$ and its spatial derivatives such that

$$
u N_{x} u \geq 0
$$

for all $u$. For simplicity it is also assumed that $N_{x} u$ is odd in $u$ and its spatial derivatives.

* Received July 5, 1973; revised version received March 5, 1975. 
The displacement $u$ satisfies the time-independent homogeneous boundary conditions

$$
\begin{array}{ll}
D_{i} u(0, t)=0, & i=1,2, \cdots, p, \\
D_{i} u(l, t)=0, & i=p+1, \cdots, 2 n
\end{array}
$$

where $D_{i}$ are linear differential operators of order $<2 n$ in $x$. Henceforth it is assumed that the time-reduced linear equation

$$
L_{x} V-\Omega^{2} V=0
$$

with $V=V(x)$ subject to

$$
\begin{aligned}
& D_{i} V(0)=0, \quad i=1,2, \cdots, p, \\
& D_{i} V(l)=0, \quad i=p+1, \cdots, 2 n
\end{aligned}
$$

forms a properly posed self-adjoint boundary value problem. It is also assumed that $L_{x}$ is positive definite and the eigenfunctions $V_{i}(x)$, corresponding to an infinite, discrete set of positive eigenvalues $\Omega_{1}{ }^{2}$ that increase monotonically to infinity, are orthonormal in the sense

$$
\int_{0}^{l} V_{i}(x) V_{i}(x) d x=\delta_{i i}
$$

and are complete in the usual sense of eigenfunction expansions [9].

In order to solve (1) the nonlinear term $N_{x} u$ is approximated by a function that is linear in $u$. To achieve this one expands $N_{x} u$ in a series of ultraspherical polynomials and then truncates the series after the linear term. The ultraspherical polynomials $P_{n}{ }^{\lambda}(v)$ are orthogonal over the interval $(-1,1)$ with respect to the weighting function $\left(1-v^{2}\right)^{\lambda-1 / 2}$ and may be obtained from Rodrique's formula [10] as

$$
P_{n}{ }^{\lambda}(v)=B_{n}{ }^{\lambda}\left(1-v^{2}\right)^{-\lambda+1 / 2}\left(\frac{d}{d v}\right)^{n}\left(1-v^{2}\right)^{n+\lambda-1 / 2}
$$

where $n$ and $\lambda, n$ being a nonnegative integer and $\lambda>-1 / 2$, are respectively the degree and the index of the polynomials. The normalization constant is given by

$$
\begin{array}{ll}
B_{n}{ }^{\lambda}=\frac{(-1)^{n} \Gamma(\lambda+1 / 2) \Gamma(n+2 \lambda)}{2^{n} n ! \Gamma(2 \lambda) \Gamma(n+\lambda+1 / 2)}, & \lambda \neq 0, \\
B_{n}{ }^{\lambda}=\frac{(-1)^{n} 2^{n} n !}{(2 n) !}, & \lambda=0 .
\end{array}
$$

Let $V_{i}(x)$ be a linear eigenfunction and $A$ be an amplitude parameter. To study nonlinear vibrations that are close to the $i$ th linear vibration, let us expand $N_{x} u$, at some $x$ where $V_{i}(x) \neq 0$, "formally" as

$$
N_{x} u=\sum_{n=0}^{\infty} C_{n} P_{n}^{\lambda}\left(u / A V_{i}\right)
$$

where the $C_{n}=C_{n}(x, \lambda, A, i)$ are given by

$$
C_{n}=\frac{\int_{-\Lambda V_{i}}^{\Lambda V_{i}} N_{x} u\left[1-\left(u / A V_{i}\right)^{2}\right]^{\lambda-1 / 2} P_{n}^{\lambda}\left(u / A V_{i}\right) d u}{\int_{-\Lambda V_{i}}^{\Lambda V_{i}}\left[1-\left(u / A V_{i}\right)^{2}\right]^{\lambda-1 / 2}\left[P_{n}^{\lambda}\left(u / A V_{i}\right)\right]^{2} d u}
$$


Note that the expansion in (9) is valid only for $\left|u / A V_{i}\right| \leq 1$. This restriction, however, can be removed by truncating the series in (9) after $n=1$, evaluating $C_{0}$ and $C_{1}$, and casting the result in a slightly different form. Let us assume that $N_{x} u$ depends only on $u$. As $N_{x} u$ is odd in $u$, one obtains immediately $C_{0}=0$. Now assume that $N_{x} u$ depends also on $u_{x}, u_{x x}, \cdots$ or even on integrals with integrands that are nonlinear in $u, u_{x}$, $u_{x x}, \cdots$. One first constructs an odd polynomial in $u$ that approximates $N_{\alpha} u$ by making use of the assumption $u \sim A V_{i}(x) \cos t$. Thus, for example,

$$
\begin{gathered}
u_{x} \sim A V_{i}{ }^{\prime} \cos t \sim\left(V_{i}^{\prime} / V_{i}\right) u, \quad u_{x x} \sim A V_{i}^{\prime \prime} \cos t \sim\left(V_{i}^{\prime \prime} / V_{i}\right) u, \cdots, \\
\int_{0}^{l} u_{x}^{2} d x \sim A^{2} \cos ^{2} t \int_{0}^{l}\left(V_{i}^{\prime}\right)^{2} d x \sim \frac{u^{2}}{V_{i}{ }^{2}} \int_{0}^{l}\left(V_{i}^{\prime}\right)^{2} d x, \cdots,
\end{gathered}
$$

and again $C_{0}=0$.

To obtain a closed-form expression for $C_{1}$, let us write

$$
N_{x} u \sim \sum_{k=0}^{\infty} \alpha_{2 k+1} u^{2 k+1}
$$

where $\alpha_{2 k+1}$ may depend on $V_{i}(x), V_{i}^{\prime}(x), \cdots$. Substituting (12) into (10) and for $n=1$, one obtains

$$
\begin{array}{ll}
C_{1}=\sum_{k=0}^{\infty} \alpha_{2 k+1} \gamma_{2 k+1}\left(A V_{i}\right)^{2 k+1} / 2 \lambda, & \lambda \neq 0, \\
C_{1}=\sum_{k=0}^{\infty} \alpha_{2 k+1} \gamma_{2 k+1}\left(A V_{i}\right)^{2 k+1}, & \lambda=0,
\end{array}
$$

where

$$
\gamma_{2 k+1}=\frac{\Gamma(k+3 / 2) \Gamma(\lambda+2)}{\Gamma(3 / 2) \Gamma(k+\lambda+2)} .
$$

Now with

$$
\begin{array}{ll}
P_{1}^{\lambda}\left(u / A V_{i}\right)=2 \lambda u / A V_{i}, & \lambda \neq 0, \\
P_{1}{ }^{0}\left(u / A V_{i}\right)=u / A V_{i}, & \lambda=0
\end{array}
$$

Eq. (9) in truncated form becomes

$$
N_{x} u \sim C_{1} P_{1}^{\lambda}\left(u / A V_{i}\right)=\sum_{k=0}^{\infty} \alpha_{2 k+1} \gamma_{2 k+1}\left(A V_{i}\right)^{2 k} u
$$

which is valid for all $\lambda>-1 / 2$. The restriction $\left|u / A V_{i}\right| \leq 1$ is removed.

The case where $V_{i}(x)=0$ at some $x$ may be regarded as a limiting case of the analysis above by letting $A V_{i} \rightarrow 0$. It is easily shown that (16) remains valid as $A V_{i}$ tends to zero and at a point where $V_{i}=0, N_{x} u$ is simply given by $\alpha_{1} u$.

With the aid of (16), defined for all $x, 0 \leq x \leq l$, Eq. (1) becomes

$$
L_{x} u+\omega^{2} u_{\imath \iota}+\sum_{k=0}^{\infty} \alpha_{2 k+1} \gamma_{2 k+1}\left[A V_{i}(x)\right]^{2 k} u=0
$$

which is linear in $u$. Seeking a $2 \pi$ time-periodic solution and expanding the spatial part of the solution in terms of the linear eigenfunctions, one writes 


$$
u(x, t)=\left(\sum_{i=1}^{\infty} A_{i} V_{i}(x)\right) \cos t .
$$

Substitution of (18) into (17) then yields

$$
\sum_{i=1}^{\infty} A_{i}\left(\Omega_{i}{ }^{2}-\omega^{2}\right) V_{i}(x)+\sum_{k=0}^{\infty} \alpha_{2 k+1} \gamma_{2 k+1}\left[A V_{i}(x)\right]^{2 k}\left(\sum_{i=1}^{\infty} A_{i} V_{i}(x)\right)=0
$$

Multiplying (19) with $V_{D}(x)$ and integrating with respect to $x$ from 0 to $l$ and using (6), one obtains

$$
A_{p}\left(\Omega_{p}{ }^{2}-\omega^{2}\right)+\int_{0}^{l}\left\{\sum_{k=0}^{\infty} \alpha_{2 k+1} \gamma_{2 k+1}\left[A V_{i}(x)\right]^{2 k}\left(\sum_{i=1}^{\infty} A_{i} V_{i}(x)\right)\right\} V_{p}(x) d x=0
$$

from which $\omega^{2}$ and $A_{p}$ will be determined below.

At this point it should be remarked that the approximations made thus far have been for the purpose of obtaining nonlinear solutions in the neighborhood of an $i$ th linear eigenvibration. The approximations are to be understood in an "asymptotic" sence that they become more and more accurate as $A$ tends to zero. In consistency with this observation, one expects that $u(x, t)$ tends to $A V_{i}(x) \cos t$ when the nonlinearity is absent. Now setting $p=i, A_{i}=A$ and keeping only the $A_{i} V_{i}(x)$ term in the series $\sum_{i=1}^{\infty} A_{i} V_{i}(x)$, one obtains

$$
\omega^{2}=\Omega_{i}{ }^{2}+\sum_{k=0}^{\infty} \gamma_{2 k+1} A^{2 k} \int_{0}^{l} \alpha_{2 k+1} V_{i}^{2 k+2}(x) d x, \quad i=1,2, \cdots,
$$

which gives the square of the $i$ th nonlinear frequency as a function of the amplitude parameter $A$. In a similar manner one may also determine all $A_{\nu}, p \neq i$, from (20).

Application. Frequency-amplitude relations for vibrating strings, beams, membranes and plates that contain nonlinear restoring forces have been obtained in [11] using the method described above. One example system is considered here as an illustration.

When axial tension is included, the dimensional equation of motion for free vibration of a uniform beam with immovable end supports may be written as [12]

$$
E I U_{X X X X}-\left(F_{0}+F\right) U_{X X}+\rho U_{T T}=0
$$

where $F_{0}$ is the initial tension and $F$ is the induced tension. $F$ is approximated as

$$
F=\frac{E S}{2 l} \int_{0}^{l}\left(U_{X}\right)^{2} d X
$$

where $S$ is the cross-sectional area and other symbols have their usual meaning. Upon introducing the dimensionless quantities

$$
\begin{aligned}
& u=U / l, \quad t=\psi T, \quad \beta=F_{0} l^{2} / E I \pi^{2}, \\
& x=(\pi / l) X, \quad \omega^{2}=\left(\rho l^{4} / E I \pi^{4}\right) \psi^{2}, \quad \epsilon=S l^{2} / 2 \pi I,
\end{aligned}
$$

one obtains, from (22), the following dimensionless equation:

$$
u_{x x x x}-\beta u_{x x}-\epsilon \int_{0}^{\pi}\left(u_{x}\right)^{2} d x u_{x x}+\omega^{2} u_{t \iota}=0
$$


which is in the form of (1) if one sets

$$
l=\pi, \quad L_{x}=\frac{\partial^{4}}{\partial x^{4}}-\beta \frac{\partial^{2}}{\partial x^{2}}, \quad N_{x} u=-\epsilon u_{x x} \int_{0}^{\pi}\left(u_{x}\right)^{2} d x .
$$

Let us consider vibrations in the first mode. By (11) and (12) one has

$$
N_{x} u=\alpha_{3} u^{3}
$$

where

$$
\alpha_{3}=-\epsilon V_{1}^{\prime \prime}(x) \int_{0}^{\pi}\left[V_{1}^{\prime}(x)\right]^{2} d x /\left[V_{1}(x)\right]^{3} .
$$

The amplitude-frequency relation now follows directly from (21) as

$$
\omega^{2}=\Omega_{1}{ }^{2}-\epsilon A^{2} \gamma_{3} \int_{0}^{\pi} V_{1}(x) V_{1}{ }^{\prime \prime}(x) d x \int_{0}^{\pi}\left[V_{1}{ }^{\prime}(x)\right]^{2} d x
$$

where

$$
\gamma_{3}=\frac{3}{2(\lambda+2)}
$$

If one defines the maximum amplitude of vibration as

$$
A^{*}=l A\left|V_{1}\right|_{\max }
$$

then the amplitude term $\epsilon A^{2}$ in (29) can be expressed in a more descriptive manner for experimental work as a ratio of $A^{*}$ and the radius of gyration $r$ for a beam of length $\pi$ as

$$
\epsilon A^{2}=\left(\frac{A^{*}}{r}\right)^{2} / 2 \pi\left|V_{1}\right|_{\max }^{2} .
$$

From Eqs. (29) and (32), along with the linear eigenfunctions [13], the frequencyamplitude relation for a simply supported beam follows as

$$
\omega^{2}=\Omega_{1}{ }^{2}+0.2500 \gamma_{3}\left(\frac{A^{*}}{r}\right)^{2}
$$

for the clamped-hinged beam

$$
\omega^{2}=\Omega_{1}{ }^{2}+0.2987 \gamma_{3}\left(\frac{A^{*}}{r}\right)^{2}
$$

and for the clamped-clamped beam

$$
\omega^{2}={\Omega_{1}}^{2}+0.3091 \gamma_{3}\left(\frac{A^{*}}{r}\right)^{2} .
$$

In Fig. 1 , the frequency ratio $\omega / \Omega_{1}$ is plotted against $\left(A^{*} / r\right)$ for clamped-clamped, clamped-supported and simply-supported beams. Two different values for $\lambda, \lambda=0$ (Tchebycheff polynomials of the first kind) and $\lambda=1 / 2$ (Legendre polynomials), are used. The results here show that the nonlinear frequencies increase with increasing amplitudes. This is to be expected as the nonlinearity included here possesses hard 


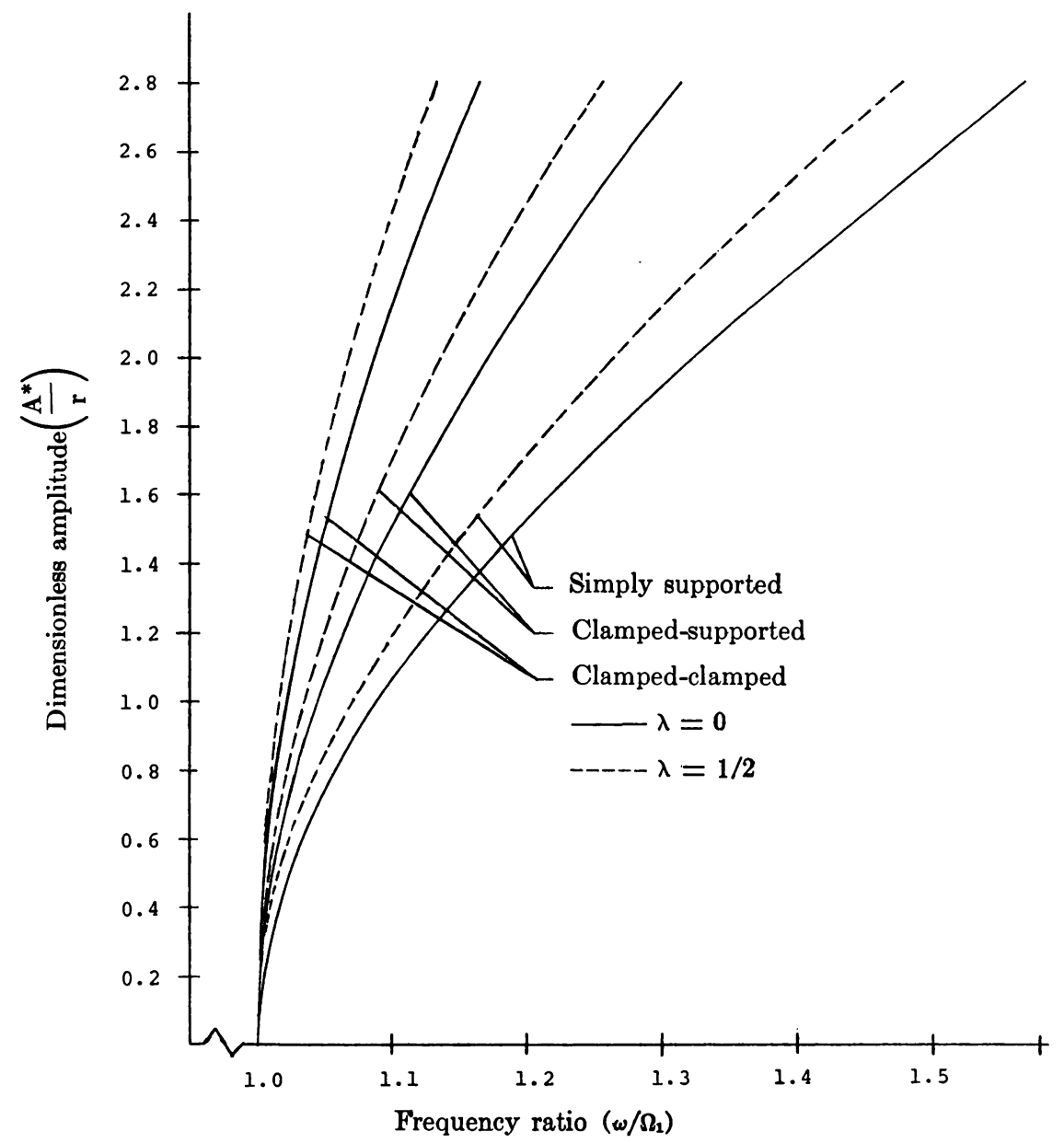

FIG. 1. Amplitude-frequency curves for beams with immovable supports.

spring characteristics as it provides additional constraints to the system. It is also interesting to note that the results here for $\lambda=0$ agree exactly with those of Evensen [14] using the perturbation method.

\section{REFERENCES}

[1] H. H. Denman and J. E. Howard, Application of ultraspherical polynomials to nonlinear oscillations I. Free oscillation of the pendulum, Quart. Appl. Math. 21, 325-330 (1964)

[2] H. H. Denman and Y. K. Liu, Applications of ultraspherical polynomials to nonlinear oscillations II. Free oscillations, Quart. Appl. Math. 22, 273-292 (1965)

[3] S. C. Sinha and P. Srinivasan, Application of ultraspherical polynomials to nonlinear autonomous systems, J. Sound Vibration 18, 55-60 (1971)

[4] S. C. Sinha and P. Srinivasan, An approximate analysis of nonlinear non-conservative systems subjected to step function excitation, J. Sound Vibration 22, 211-219 (1972)

[5] R. M. Garde, Application of Gegenbauer polynomials to nonlinear damped oscillations, J. Science Engineering Res. 11, 157-166 (1967)

[6] M. L. Adelberg and H. H. Denman, Phase plane analysis of nonlinear systems using weighted linearization, Int. J. Nonlinear Mech. 4, 311-324 (1969) 
[7] S. C. Sinha and P. Srinivasan, A weighted mean square method of linearization in nonlinear oscillations, J. Sound Vibration 16, 139-148 (1971)

[8] L. K. Liu, Application of ultraspherical polynomial approximation to nonlinear systems with two degrees of freedom, Ph.D. Dissertation, Wayne State University, 1965

[9] R. Courant and I. Hilbert, Methods of mathematical physics, Vol. I, Interscience Publishers, New York, 1966

[10] E. D. Rainville, Special functions, The MacMillan Co., New York, 1960

[11] P. T. Blotter, Free periodic vibrations of continuous systems governed by nonlinear partial differential equations, Ph.D. Dissertation, Michigan State University, 1968

[12] S. Woinowsky-Krieger, The effect of an axial force on the vibration of hinged bars, J. Appl. Mech. $17,35-36(1950)$

[13] E. Volterra and E. C. Zachmanoglou, Dymanics of vibrations, Merrill Books, Inc., Columbus, Ohio, 1965.

[14] D. A. Evensen, Nonlinear vibrations of beams with various boundary conditions, AIAA J. 6, 370-372 (1968) 